\title{
Bacteriological study of blood stream infection (BSI) in ICU patients
}

\author{
Ullas Bhabhor ${ }^{1}$, Hitesh Bhabhor ${ }^{2, *}$, Hitesh Shingala ${ }^{3}$, Mala Sinha ${ }^{4}$ \\ ${ }^{1}$ Assistant Professor, Stem Cell Therapy Institute, Government Medical College, New Civil Hospital, Surat, Gujarat, ${ }^{2}$ Tutor, \\ Dept. of Community Medicine, Government Medical College, New Civil Hospital, Surat, Gujarat, ${ }^{3}$ Associate Professor, \\ ${ }^{4}$ Professor and Head, Dept. of Microbiology, G.G. Hospital, Jamnagar, Gujarat, India
}

*Corresponding Author:

Email: hitesh1357@yahoo.co.in

Received: $2^{\text {nd }}$ May, 2018

Accepted: $31^{\text {th }}$ July, 2018

\begin{abstract}
Introduction: Blood stream infection is a most common health care associated infection causes significant disease and death worldwide. Patients are admitted to ICUs carry higher risk of nosocomial BSI than those who are admitted in other type of units. Early Identification of causative agents $\&$ their antibiotic sensitivity pattern are useful to reduce mortality \& improve clinical outcome of the patients.

Materials and Methods: A prospective study conducted in tertiary care hospital. During study all the patients monitor for nosocomial blood stream \infection. Blood sample collected from suspected patient of blood stream infection for detection causative organism \& antimicrobial susceptibility pattern.

Result: A Total 620 samples were tested out of these 173 (27.90\%) were positive growth in culture. Maximum number of blood stream infections were from paediatric ICU (69.62\%), followed by Medical ICU (18.35\%) and from surgical ICU (12.02\%). In present study incidence of length of stay is more after five day of admission in ICU (68.35\%). The incidence of blood stream infection due to gram positive organism was (51.28\%). Among them Staphylococcus aureus 72 (45.56\%) was most common organism. Gram negative organism were quite low (48.73\%) among them Klebsiella spp. (16.45\%) was most common. The antimicrobial susceptibility pattern of isolated organisms showed high resistance to routinely used antimicrobial agents.

Conclusion: The systemic approach by studying the culture of organisms from the foci and blood culture and the antibiotic profile may help the clinician to select appropriate empirical antimicrobial agents.
\end{abstract}

Keywords: Antimicrobial sensitivity, Causative organisms, Intensive care Units, Nosocomial blood stream infection.

\section{Introduction}

The importance of ICU is inevitable in the control of infection and treatment of the most variable and severe illness of the human body. ${ }^{1}$ Critically ill patients in ICU are at high risk of getting the hospital Acquired infections, as evidence by several studies. The risk of acquiring Nosocomial infections are increase up to 5 to 10 times in ICU patients where it is compared to patient from general ward. ${ }^{2}$

Nosocomial infections become evident clinically after $48 \mathrm{hrs}$ of hospitalization and it is not originate from patient's original admitting diagnosis. These types of infections cause significant morbidity, mortality and have a considerable impact on health care associated costs. $^{3}$

The rate of Nosocomial infections in the ICU is increase worldwide mainly due to use of invasive procedures performed in the ICU. The therapeutic interventions are related with infectious complications such as sophisticated life support, intravenous fluid therapy, prosthetic devices, indwelling catheters, immunosuppressive therapy, changes in the population at high risk and the use of broad spectrum antimicrobial agents leading to multidrug resistance organisms, they contributed to the evolution of the Nosocomial infections. ${ }^{4}$

Out of all types of Nosocomial infections blood stream infection is a serious health problem in all over the world. ${ }^{3}$ Blood stream infection (BSI) is an important event which is responsible for longest hospital stay. Two types BSIs can be occur. It may be healthcare associated or community-associated infection. Health-care associated blood stream infection is a frequently occurring in hospitalised patients. Blood stream infections continue can be severe \& always lifethreatening condition. Emerging advanced life-support facilities and development of newer antimicrobial agents are associated with high BSIs mortality rate ranging $25-50 \% .^{5}$

Gram-negative organisms are the leading cause of blood stream infections. ${ }^{5}$ Approximately $75 \%$ of primary Bloodstream infections is caused by gramnegative bacilli due to the development of resistance against $\beta$-lactam agents like ESBL (Extended Spectrum Beta Lactamase). Klebsiella pneumonia, Escherichia coli, Proteus spp, Pseudomonas spp., Enterobacter spp., Citrobacter spp., and Acinetobacter spp.. are the common.

\section{Materials and Methods}

This is a prospective study in tertiary care hospital. Samples are collected from different ICUs (Paediatric ICUs, medical and surgical ICU) at Tertiary care Hospital, patient having at least one of the following criteria: Patient has a recognized pathogen cultured from one or more blood cultures and the organism 
cultured from blood is not related to an infection at another site or Patient has at least one signs or symptoms like raise respiratory rate, abnormally rise heart rate, low or high blood pressure, fever $\left(>38^{\circ} \mathrm{C}\right)$ or hypothermia, rigors, chills, severe local infections (pneumonia, endocarditis, intra-abdominal suppuration, pyelonephritis, meningitis, etc...) or At least one of the Common skin contaminant (e.g, Bacillus sp., micrococci, Coagulase-negative staphylococci or Diphtheroids ) is isolated from two or more blood cultures collection on separate occasions.

The Signs and symptoms of nosocomial infection appear after 48 hours of hospital admission, and there are no any signs and symptoms of infection at the time of admission confirm by history and clinical examination of patient. Blood was collected from clinically suspected cases blood stream infection following strict aseptic precautions. Withdraw $5 \mathrm{ml}$ of an adult or about $2 \mathrm{ml}$ from a young child Blood was inoculated aseptically into $50 \mathrm{ml}$ (for adult) \& $20 \mathrm{ml}$ (for paediatric) Glucose broth (1:10 ratio). After that culture bottles were incubated at $37^{\circ} \mathrm{C}$ aerobically in incubator and periodic subcultures done in solid media like, MacConkey agar media, blood agar media and chocolate agar media after overnight incubation on day 1 , day 3, for isolation. The growth obtained was identified by conventional biochemical test. ${ }^{6}$ And isolated organism's Antibiotic Susceptibility Testing was done by Kirby-Bauer disk diffusion method by using Mueller Hinton agar plate. The turbidity of broth containing test organism compare with $0.5 \mathrm{McF}$ arland standard and it inoculate a Lawn culture on a Muller Hinton agar plate The inoculated plated was allowed to dry for few minutes in room temperature with lid closed and then the appropriate antibiotic discs were placed on the agar surface with the sterile forceps and pressed gently to make sure that the disc was in even contact with the medium. The discs were placed in such a way that they were $15 \mathrm{~mm}$ away from the edge of the plate and the distance between each disc was not less than $25 \mathrm{~mm}$. Only 6 discs were placed per petri plate then plates were incubated at $37^{\circ} \mathrm{C}$ aerobically overnight. Interpretation of result measured as susceptible, Intermediate or resistant identified by measuring diameter of the zones as per as per CLSI guidelines. Utilisation of drug are An-amikacin, g - gentamycin, $\mathrm{ba} / \mathrm{ct}$ - co-trimoxazole, cip / rc - ciprofloxacin, ctx ceftriaxone, cf - cefotaxime, $\mathrm{nr}$ - norfloxacin, slb/as ampicillin/sulbactam, pc - piperacillin, ch chloramphenicol, ci - ceftizoxime, zn - ofloxacine, pr cephalexin, az - azithromycin, lz -linezoid, cx cloxacillin, te - tetracycline

\section{Result}

Total 620 samples were collected from suspected blood stream infection from different ICUs in Tertiary care Hospital. Out of 620 samples were obtained from these $173(27.90 \%)$ were positive growth in culture, while 447 (72.09\%) samples recommended as no growth in culture. sex wise distribution of positive samples in that $94(54.33 \%)$ patients were male, while $79(45.66 \%)$ were female. age wise distribution of cases shows 1 month-15 years children were 123 (71.09\%), 16-30 years patients were $13(7.51 \%), 31-45$ years patients were $24(13.87 \%), 46-60$ years were 08 $(4.62 \%)$ and above 60 years were 05 (2.89\%). ward wise distribution of cases shows in medical ICU cases were $29(16.76 \%)$ and surgical ICU cases were 21 $(12.13 \%)$ while maximum cases observed in paediatric ICU were $123(71.09 \%)$. Incidence of length of stay in< 5 days were $56(32.36 \%)$ while in $\geq 5$ days rate were $117(67.63 \%)$ in present study showing nosocomial blood stream infection were $96(55.49 \%)$ by Gram positive organisms while $77(44.50 \%)$ by Gram negative organisms. Fig. 1 shows isolated gram positive organism \& Fig. 2 shows isolated gram negative organisms in present study. In Fig. 3 shows Antibiotic sensitivity of gram positive organisms \& Fig. 4 shows Antibiotic sensitivity of gram negative organisms.

\section{Discussion}

Bacteria are champions of evolution, and a few microbes have adapted to a point where they pose serious clinical challenges for humans. In present study rate of blood stream infection is $27.90 \%$ comparable with different study of $22.68 \%$ in Jan Muhammad Shaikh et al. 2008, ${ }^{7} 27 \%$ in Rizvi M et al. 20078, 29.2\% in Stephane Hugonnet et al.20049, 34.7\% in Jordi valles et al. 2011. ${ }^{10}$ In present study, the sex-wise prevalence of BSI are more in male $94(54.33 \%)$ than female $79(45.66 \%)$. This is comparable with study of Jan Muhammad Shaikh et $\mathrm{al}^{7}$ (M-52.6\% \& F-47.4\%), Christine Bonnal et al. ${ }^{11}$ (M-60\% \& F-39.70\%), Prowle et al. $^{12}$ (M-65.2\% \& F-34.84\%). In present study, the age-wise prevalence of BSI are more in paediatric patient $123(71.09 \%)$ which was comparable with Shahla latif et al. ${ }^{13}(76 \%)$, while adult patient it was more common in age group of 31 to $45 \mathrm{yrs}$ (13.92\%) which was comparable with the study of Olgica Gajović et $\mathrm{al}^{14}$ (mean age 40yrs) and in Dr. Venu Gopal et al. ${ }^{15}$ (mean age 47), in present study, maximum BSI diagnosed patients were from Paediatric ICUs 123 (71.09\%), where paediatric ICUs comparable with study of Shahla Latif et al. ${ }^{13}(76 \%)$. In present study, incidence of length of stay was higher in $\geq 5$ days $(67.63 \%)$, which was comparable with study of Stéphane Hugonnet et $\mathrm{al}^{9}$ (mean day-5 day) Ambanaa Gowda Durgad et al (mean day-6.42). In present study $55.49 \%$ rate of gram positive organisms were comparable with the $(50 \%)$ rate in study of Eric A.J. Hoste et al $^{16}$ while $44.50 \%$ rate of gram negative organisms comparable with the $(39.7 \%)$ rate in study of Eric A.J. Hoste et $\mathrm{al}^{16}$ and Jordi Vallés et $\mathrm{al}^{10}(42.2 \%)$. Table 1 shows rate of isolated gram positive organisms comparable with different studies table 2 shows rate of isolated gram negative organisms comparable with 
different studies. In gram positive organism S.aureus were more sensitive to Linezoid \& Ampicilin/Sulbactam followed by Cloxacillin then CoTrimoxazole \& Tetracycline, Cephalexin Which are comparable with similar study of R.B. Patwardhan et al, ${ }^{17}$ Yahiya A. Abass et al, ${ }^{18}$ Easow JM et al, ${ }^{5}$ Sunit Singhi et al, ${ }^{19}$ while isolated Enterococci most sensitive to Tetracycline (100\%), Linezoid, Amikacin, Gentamycin (66.66\%), Ciprofloxacin, Co-Trimoxazole, Ampicilin/Sulbactam, Cephalexin, Azithromycin, Cloxacillin (33.33\%) while resistance to Cefotaxime \& isolated Streptococcus spp. most sensitive to Linezoid(100\%), Tetracycline, followed by Ampicilin/Sulbactam \& Cefotaxime \& Cephalexin, Ciprofloxacin, Gentamycin, Co-Trimoxazole, Gentamycin, Cloxacillin (50\%) Amikacin, Azithromycin $(16.66 \%)$ sensitivity pattern of all gram positive organism are comparable with similar study of R.B. Patwardhan et al,${ }^{17}$ Yahiya A. Abass et al,,${ }^{18}$ Easow JM et al,.5 In present study, Klebsiella were most sensitive to Amikacin, Ciprofloxacin, Gentamycin followed by Chloramphenicol, Tetracycline, Ofloxacine Isolated Pseudomonas were highly sensitive to CoTrimoxazole followed by Ciprofloxacin, Cefotaxime \& Ofloxacine. Acinatobacter spp. were sensitive to Ofloxacine \& Amikacin followed by Gentamycin, CoTrimoxazole \& Tetracycline. E.coli were most sensitive to Amikacin, Gentamycin, Co-Trimoxazole, Ampicillin/Sulbactam. Proteous spp. \& Providencia spp were sensitive to all drugs. Sensitivity pattern of all gram negative organism, R.B. Patwardhan et al, ${ }^{17} \mathrm{~S}$ sager Faiz et al. ${ }^{21}$ US Kamat et al, ${ }^{22}$ Olgica Gajović et al. $^{14}$

Table 1: Common gram positive organism found in different studies

\begin{tabular}{|c|c|c|c|c|}
\hline \multirow[t]{2}{*}{ Various studies } & \multicolumn{4}{|c|}{ Organism isolated\% } \\
\hline & S.aureus & Enterococci & Streptococus spp. & CONS \\
\hline Shaaban H. Ahmed et $\mathrm{al}^{3} 2009$ & $29.2 \%$ & $7.4 \%$ & $2.3 \%$ & - \\
\hline Rosineide M et al ${ }^{23} 2007$ & $49.5 \%$ & $7.1 \%$ & - & - \\
\hline $\mathrm{N}$ Nigatu Endaiafer et $\mathrm{al}^{20} .2008$ & $37.5 \%$ & - & $12.4 \%$ & - \\
\hline Chen $\mathrm{R}$ et $\mathrm{al}^{24} 2012$ & $28.0 \%$ & - & - & - \\
\hline Hilmer Wisplinghoff et al ${ }^{25} 2004$ & $10.3 \%$ & $9.4 \%$ & - & - \\
\hline Outi Lyytikäinen et al ${ }^{26} 2004$ & - & - & $5 \%$ & - \\
\hline David K. Warren et al ${ }^{27} 2001$ & $8 \%$ & $21 \%$ & $3 \%$ & - \\
\hline Silvia Natoli et al $^{28} 2009$ & - & - & - & $13 \%$ \\
\hline M.Mer ${ }^{29} 2005$ & - & - & - & $11 \%$ \\
\hline Easow JM et al ${ }^{5} 2010$ & - & $2.1 \%$ & - & - \\
\hline Present study & $41.61 \%$ & $1.73 \%$ & $3.46 \%$ & $8.67 \%$ \\
\hline
\end{tabular}

Table 2: Common gram negative organism found in different studies

\begin{tabular}{|c|c|c|c|c|c|c|}
\hline \multirow[t]{2}{*}{ Various studies } & \multicolumn{6}{|c|}{ Organism isolated \% } \\
\hline & 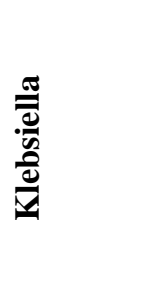 & 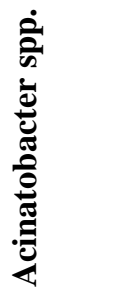 & 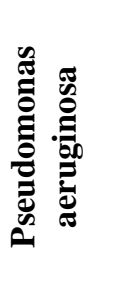 & 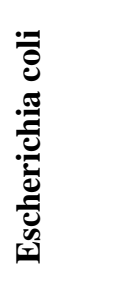 & 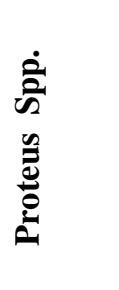 & 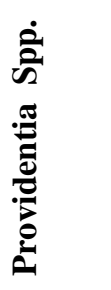 \\
\hline Dr. Venu Gopal L. ${ }^{15} 2008$ & $13.15 \%$ & $2.23 \%$ & $15.78 \%$ & $31.57 \%$ & $2.63 \%$ & - \\
\hline Stijn Blot et. al. ${ }^{30} 2002$ & $15.9 \%$ & - & $18.8 \%$ & $30.8 \%$ & - & - \\
\hline Svenja J et al..$^{31} 2006$ & $24.3 \%$ & - & $14.8 \%$ & $19.9 \%$ & - & - \\
\hline Olgica Gajović et al ${ }^{14}, 2011$ & $23.3 \%$ & $12.3 \%$ & $30.3 \%$ & $15.8 \%$ & $16.9 \%$ & $1.9 \%$ \\
\hline Didier Pittet et al ${ }^{32} 1997$ & $6.2 \%$ & - & $5.1 \%$ & $7.9 \%$ & - & - \\
\hline Blot $S$ et $\mathrm{al}^{33} 2003$ & - & $7.8 \%$ & - & - & - & - \\
\hline Easow JM et al ${ }^{5} 2010$ & $15.62 \%$ & $4.2 \%$ & $8.3 \%$ & $10.4 \%$ & - & - \\
\hline Present study & $15.02 \%$ & $9.82 \%$ & $9.82 \%$ & $8.09 \%$ & $1.15 \%$ & $0.57 \%$ \\
\hline
\end{tabular}




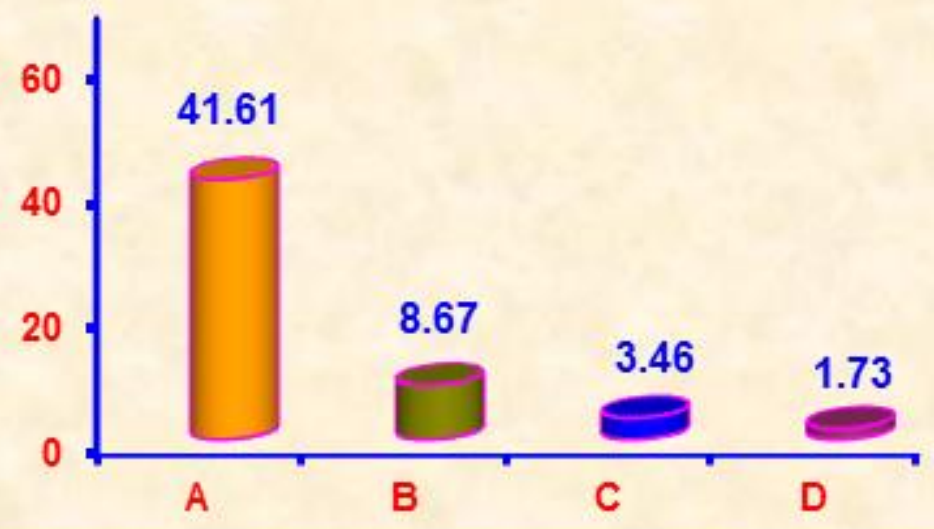

Fig. 1: Graph shows isolated gram positive organism isolated

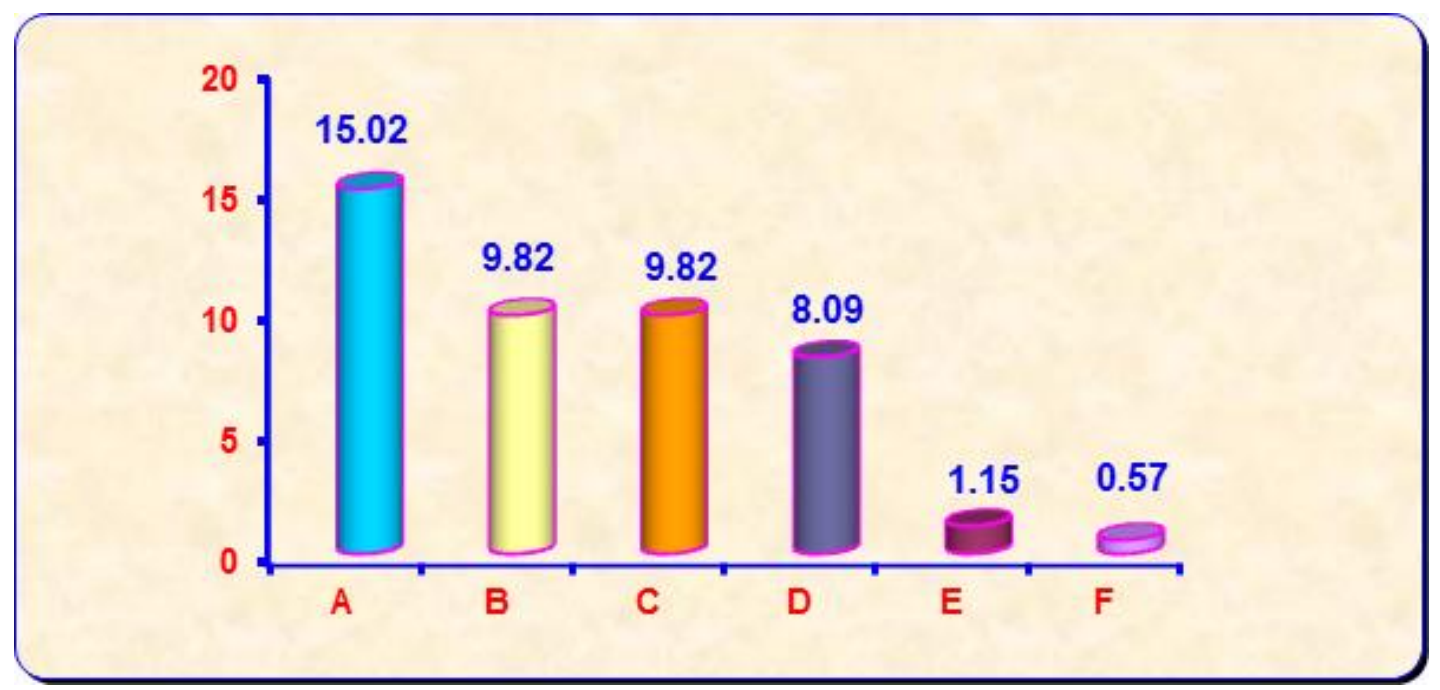

Fig. 2: Graph shows isolated gram negative organism isolated

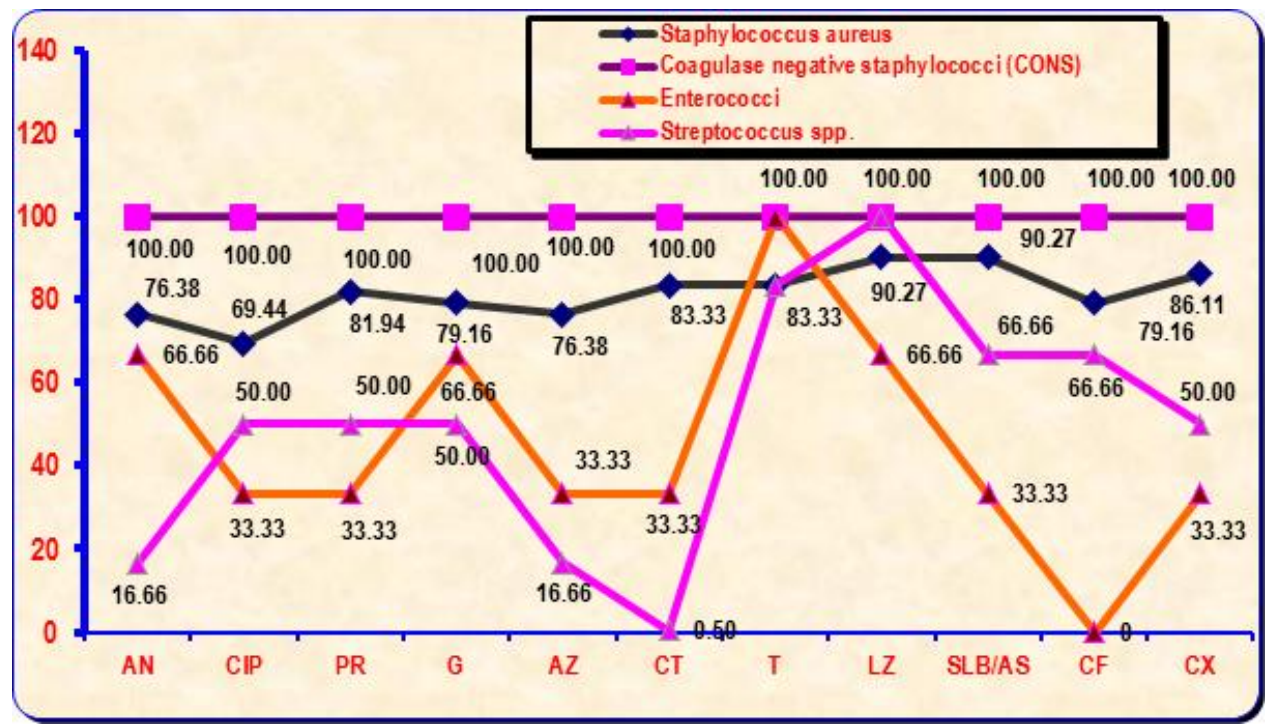

Fig. 3 


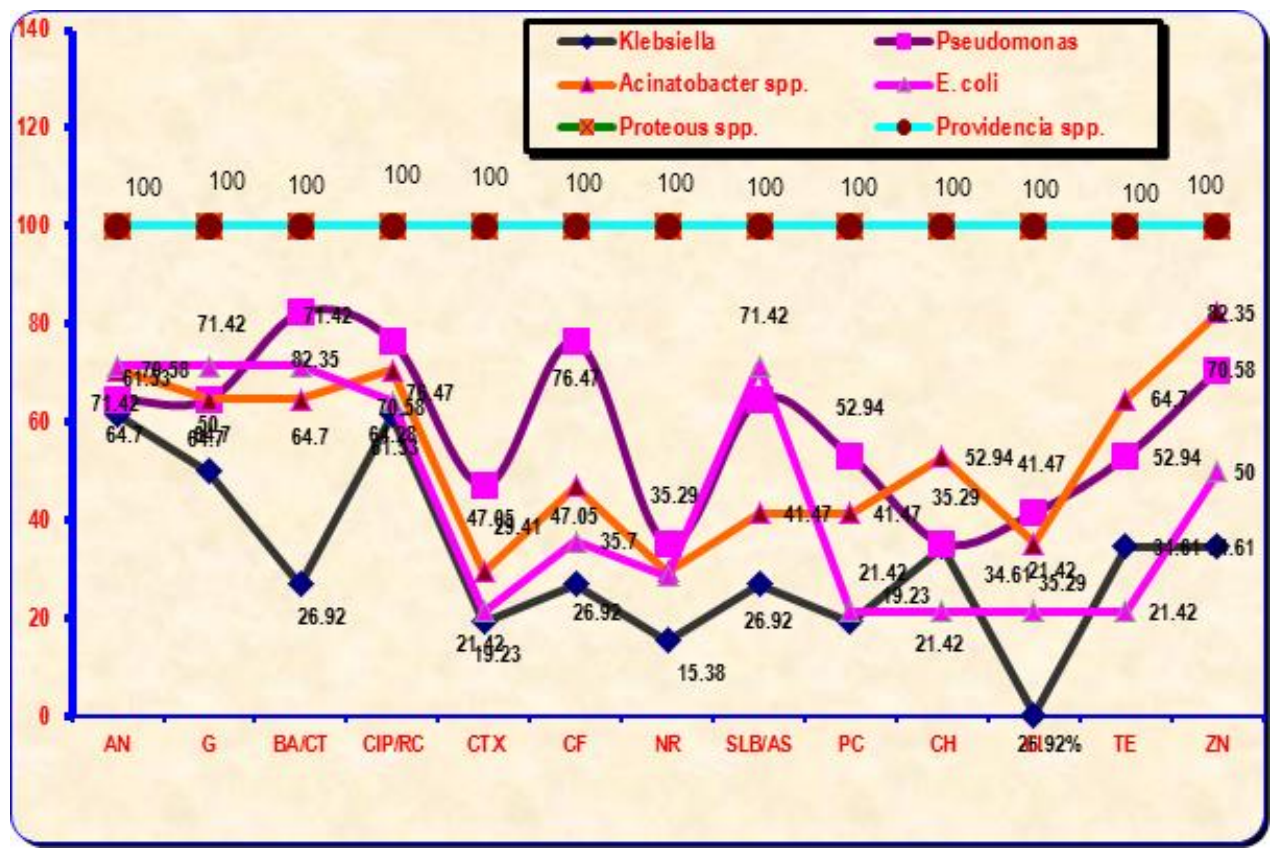

Fig. 1

\section{Conclusion}

Paediatric patient are more prone to hospital acquired infections \& it cause of morbidity and mortality due to various risk factors. The epidemiology of gram-positive bacteraemia is changing and requires appropriate management and treatment strategies to maximize patient outcome. The systemic approach by studying the culture of organisms from the foci and blood culture and the antibiotic profile may help the clinician to select appropriate empirical antibiotic. Moreover stringent hospital infection control measures and a good antibiotic policy for the hospital is the need of the hour.

\section{Reference}

1. Hassanzadeh P, Motamedifar M, Hadi N. Prevalent bacterial infections in intensive care units of Shiraz University of medical sciences teaching hospitals, Shiraz, Iran. Jpn J Infect Dis. 2009;62(4):249-53.

2. Dwivedi M, Mishra A, Singh RK, Azim A, Baronia AK, Prasad KN. Nosocomial cross-transmission of Pseudomonas aeruginosa between patients in a tertiary intensive care unit. Indian Journal of Pathology and Microbiology. 2009;52(4):509.

3. Ahmed SH, Daef EA, Badary MS, Mahmoud MA, AbdElsayed AA. Nosocomial blood stream infection in intensive care units at Assiut University Hospitals (Upper Egypt) with special reference to extended spectrum $\beta$ lactamase producing organisms. BMC research notes. 2009;2(1):76.

4. Shalini S, Kranthi K, Gopalkrishna BK. The microbiological profile of nosocomial infections in the intensive care unit. J Clin Diagn Res. 2010;(5):3109-12.

5. Easow JM, Joseph NM, Dhungel BA, Chapagain B, Shivananda PG. Blood stream infections among febrile patients attending a teaching hospital in Western Region of Nepal. Australasian Medical Journal (Online). 2010;3(10):633.
6. Collee JG, Duguid JP, Fraser AG, Marmion BP, Simmons A. Laboratory strategy in the diagnosis of infective syndromes. Mackie and McCartney practical medical microbiology. 1996;14:53-94.

7. Shaikh JM, Devrajani BR, Shah SZ, Akhund T, Bibi I. Frequency, pattern and etiology of nosocomial infection in intensive care unit: an experience at a tertiary care hospital. J Ayub Med Coll Abbottabad. 2008;20(4):37-40.

8. Rizvi MF, Hasan Y, Memon AR, Abdullah M, Saleem S, Shakeel J. Pattern of nosocomial infection in two intensive care units of a tertiary care hospital in Karachi. Journal of the College of Physicians and Surgeons-Pakistan: JCPSP. 2007;17(3):136-9.

9. Hugonnet S, Sax H, Eggimann P, Chevrolet JC, Pittet D. Nosocomial bloodstream infection and clinical sepsis. Emerging infectious diseases. 2004;10(1):76.

10. Valles J, Rello J, Ochagavía A, Garnacho J, Alcalá MA. Community-acquired bloodstream infection in critically ill adult patients: impact of shock and inappropriate antibiotic therapy on survival. Chest. 2003;123(5):1615 24.

11. Bonnal C, Mourvillier B, Bronchard R, de Paula D, Armand-Lefevre L, L'heriteau F, Quenon JL, Lucet JC. Prospective assessment of hospital-acquired bloosdstream infections: how many may be preventable?. Qual Saf Health Care. 2010;19(5):e30.

12. Prowle JR, Echeverri JE, Ligabo EV, Sherry N, Taori GC, Crozier TM, et al.. Acquired bloodstream infection in the intensive care unit: incidence and attributable mortality. Critical Care. 2011;15(2):R100.

13. Latif SH, Anwar MS, Ahmad IS. Bacterial pathogens responsible for blood stream infection (BSI) and pattern of drug resistance in a tertiary care hospital of Lahore. Biomedica. 2009;25(2):101-5.

14. Gajović O, Tomović M, Stanarcić J, Canović P, Todorović Z, Lazić Z. Clinical characteristics of nosocomial infections of patients with acute central nervous system infections treated in ICU. Medicinski Glasnik. 2011;8(2).

15. Venu Gopal L. A Study of foci of infection on admission in patients with sepsis in Multidisciplinary Intensive Care Unit (Doctoral dissertation). 
16. Hoste EA, Blot SI, Lameire NH, Vanholder RC, De Bacquer D, Colardyn FA. Effect of nosocomial bloodstream infection on the outcome of critically ill patients with acute renal failure treated with renal replacement therapy. Journal of the American Society of Nephrology. 2004;15(2):454-62.

17. Patwardhan RB, Dhakephalkar PK, Niphadkar KB, Chopade BA. A study on nosocomial pathogens in ICU with special reference to multiresistant Acinetobacter baumannii harbouring multiple plasmids. Indian Journal of Medical Research. 2008;128(2):178.

18. Abass YA. Blood Stream Infections in Al-Nassyria City. AL-TAQANI. 2009;22(2):76-80.

19. Singhi S, Ray P, Mathew JL, Jayashree M. Nosocomial bloodstream infection in a pediatric intensive care unit. The Indian Journal of Pediatrics. 2008;75(1):25-30.

20. Endalafer N, Gebre-Selassie S, Kotiso B. Nosocomial bacterial infections in a tertiary hospital in Ethiopia. Journal of Infection Prevention. 2011;12(1):38-43.

21. Saghir S, Faiz M, Saleem M, Younus A, Aziz H. Characterization and anti-microbial susceptibility of gram-negative bacteria isolated from bloodstream infections of cancer patients on chemotherapy in Pakistan. Indian journal of medical microbiology. 2009;27(4):341.

22. Kamat US, Ferreira AM, Savio R, Motghare DD. Antimicrobial resistance among nosocomial isolates in a teaching hospital in Goa. Indian journal of community medicine: official publication of Indian Association of Preventive \& Social Medicine. 2008;33(2):89.

23. Ribas RM, Freitas C, Gontijo Filho PP. Nosocomial bloodstream infections: organisms, risk factors and resistant phenotypes in the Brazilian University Hospital. Brazilian Journal of Infectious Diseases. 2007;11(3):3514.

24. Chen R, Yan ZQ, Feng D, Luo YP, Wang LL, Shen DX. Nosocomial bloodstream infection in patients caused by Staphylococcus aureus: drug susceptibility, outcome, and risk factors for hospital mortality. Chinese medical journal. 2012;125(2):226-9.

25. Wisplinghoff H, Bischoff T, Tallent SM, Seifert H, Wenzel RP, Edmond MB. Nosocomial bloodstream infections in US hospitals: analysis of 24,179 cases from a prospective nationwide surveillance study. Clinical infectious diseases. 2004;39(3):309-17.
26. Lyytikäinen O, Rautio M, Carlson P, Anttila VJ, Vuento R, Sarkkinen H, Kostiala A, Väisänen ML, Kanervo A, Ruutu P. Nosocomial bloodstream infections due to viridans streptococci in haematological and nonhaematological patients: species distribution and antimicrobial resistance. Journal of Antimicrobial Chemotherapy. 2004;53(4):631-4.

27. Warren DK, Zack JE, Elward AM, Cox MJ, Fraser VJ. Nosocomial primary bloodstream infections in intensive care unit patients in a nonteaching community medical center: a 21-month prospective study. Clinical infectious diseases. 2001;33(8):1329-35.

28. Natoli S, Fontana C, Favaro M, Bergamini A, Testore GP, Minelli S, Bossa MC, Casapulla M, Broglio G, Beltrame A, Cudillo L. Characterization of coagulasenegative staphylococcal isolates from blood with reduced susceptibility to glycopeptides and therapeutic options. BMC infectious diseases. 2009;9(1):83.

29. Mer M. Nosocomial bloodstream infection. Southern African Journal of Epidemiology and Infection. 2005;20(2):61-2.

30. Blot S, Vandewoude K, De Bacquer D, Colardyn F. Nosocomial bacteremia caused by antibiotic-resistant gram-negative bacteria in critically ill patients: clinical outcome and length of hospitalization. Clinical infectious diseases. 2002;34(12):1600-6.

31. Albrecht SJ, Fishman NO, Kitchen J, Nachamkin I, Bilker WB, Hoegg C, Samel C, Barbagallo S, Arentzen J, Lautenbach E. Reemergence of gram-negative health care-associated bloodstream infections. Archives of internal medicine. 2006;166(12):1289-94.

32. Pittet D, Li N, Woolson RF, Wenzel RP. Microbiological factors influencing the outcome of nosocomial bloodstream infections: a 6-year validated, populationbased model. Clinical infectious diseases. 1997;24(6):1068-78.

33. Blot S, Vandewoude K, Colardyn F. Nosocomial bacteremia involving Acinetobacter baumannii in critically ill patients: a matched cohort study. Intensive care medicine. 2003;29(3):471-5.

How to cite this article: Bhabhor $\mathrm{U}$, Bhabhor $\mathrm{H}$, Shingala H, Sinha M. Bacteriological study of blood stream infection (BSI) in ICU patients. Indian J Microbiol Res. 2018;5(3):368-373. 